\title{
A simple approach to a curved hexaquinane
}

\author{
Sambasivarao Kotha* and Sivakumar Rallapalli \\ Department of Chemistry, Indian Instiute of Technology, Powai, Mumbai-400076, India \\ E-mail: srk@chem.iitb.ac.in
}

This article is dedicated to Prof. James Cook on the occasion of his $65^{\text {th }}$ Birthday

\begin{abstract}
The conversion of tetraquinane 2 to the unknown curved $\mathrm{C}_{20}$-hexacyclic dione $\mathbf{3}$ is described utilizing the cyclopentane annulation strategy.
\end{abstract}

Keywords: Cyclopentane annulation, Tetraquinane, Hexaquinane

\section{Introduction}

In our attempts directed towards the synthesis of tetraquinanes and hexaquinanes, ${ }^{1,2}$ we observed the formation of tetraquinane $\mathbf{2}^{1}$ in small amounts. This article describes our efforts to convert the tetracyclic intermediate 2 into the unknown curved $\mathrm{C}_{20}$-hexacyclic dione 3 (Scheme 1).

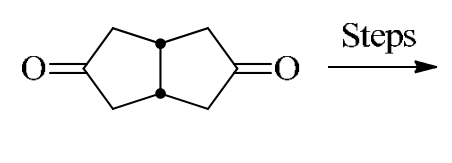

1

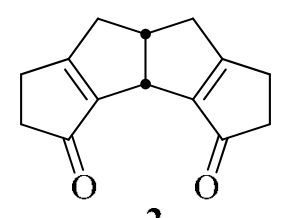

2

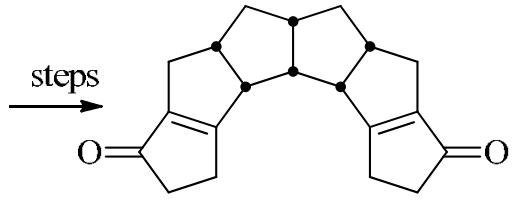

3

\section{Scheme 1}

\section{Results and Discussion}

The starting material, bicyclo[3.3.0]octane 3,7-dione 1, was obtained in a three step sequence using the Weiss-Cook reaction. ${ }^{3}$ Cyclopentane annulation of $\mathbf{1}$ provided the tetracyclic intermediate 2 as a minor product. ${ }^{1}$ Upon reduction with hydrogen in presence of $10 \% \mathrm{Pd} / \mathrm{C}$ at 3 atm, the tetracyclic intermediate 2 gave a mixture of tetracyclic diones $\mathbf{4}$ and $\mathbf{5}$ in the ratio 1:4 in $76 \%$ yield (Scheme 2). The difference in the ratio can be attributed to the stability of cis-syn-cis- 
syn-cis-fusion in isomer 5 in comparison with the cis-syn-cis-anti-cis-fused isomer $\mathbf{4}$. The isomeric diones $\mathbf{4}$ and $\mathbf{5}$ were separated by column chromatography, and structural assignments are based on spectral evaluations. The minor isomer $\mathbf{4}$ exhibits fourteen resonance lines in the proton-decoupled ${ }^{13} \mathrm{C}$ NMR spectrum, which confirms its unsymmetrical nature, while the major isomer 5 shows eight lines consistent with the mirror plane symmetry in the molecule.

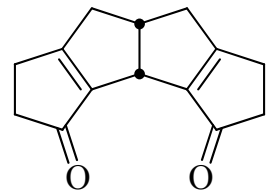

2

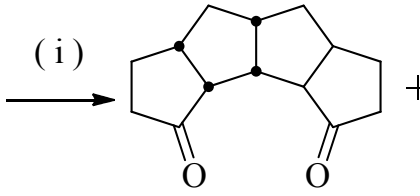

4

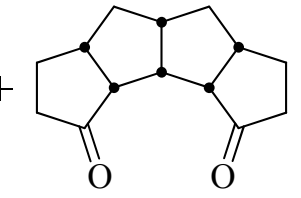

5

Scheme 2. (i) $\mathrm{H}_{2}, 10 \%$ Pd-C, EtOAc, 3 atm.

Upon reaction with allylmagnesium bromide, the tetracyclic dione $\mathbf{5}$ furnished diol $\mathbf{6}$ and the trans-annular product 7 (45\% and 30\%, respectively; Scheme 3). In agreement with the proposed structures, the proton decoupled ${ }^{13} \mathrm{C}$ NMR spectrum of 6 shows 11 lines; 7 exhibits well-resolved resonances for 17 carbon atoms, and the structure was further confirmed by X-ray diffraction studies. ${ }^{4}$ Formation of $\mathbf{7}$ is envisaged by the transformation of one carbonyl group of $\mathbf{5}$ into an enolate, which reacts in a trans-annular manner with the other carbonyl group. The resulting ketol reacts with a second molecule of the Grignard reagent to produce the monoallylic diol 7. The symmetrical diallylic diol $\mathbf{6}$ and the monoallylic diol 7 were converted into the corresponding lactones $\mathbf{8}$ and $\mathbf{9}$ by hydroboration-oxidation sequences. Methanesulphonic acid/ $\mathrm{P}_{2} \mathrm{O}_{5}$ caused the symmetrical lactone $\mathbf{8}$ to rearrange and form the curved hexacyclic enone 3.
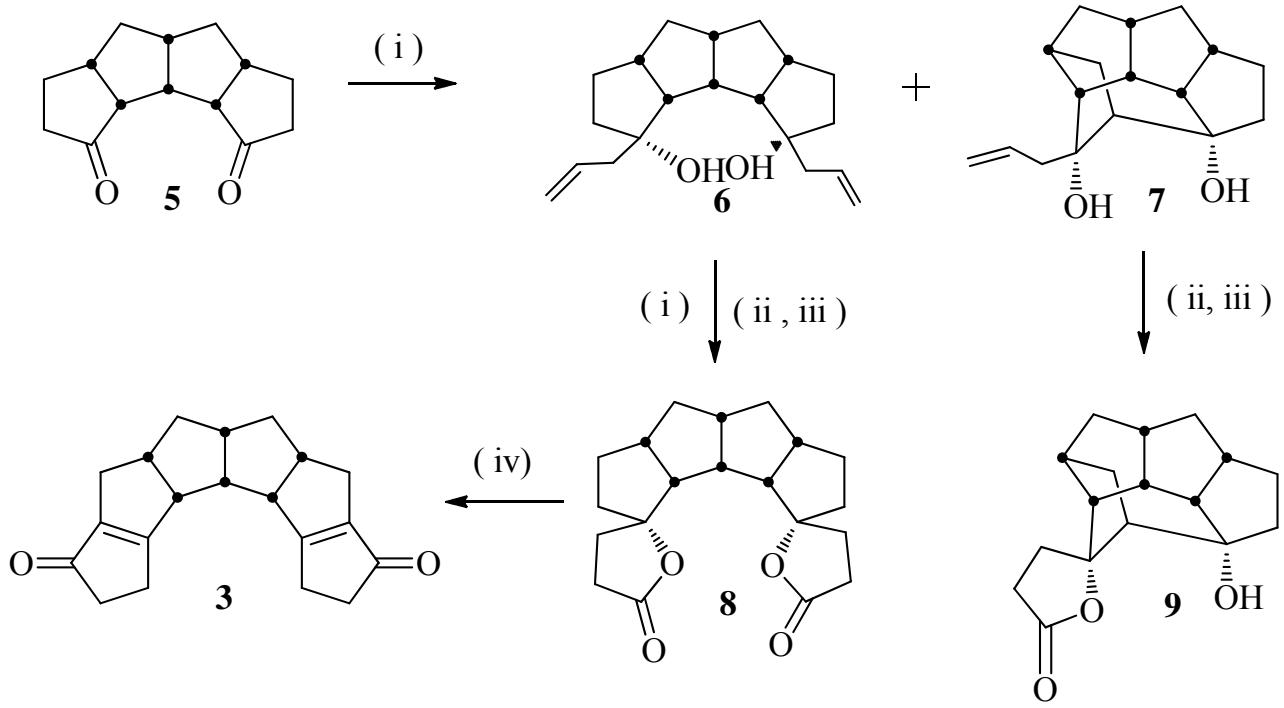

Scheme 3. (i) Allylmagnesium bromide/ether, THF. (ii) $\mathrm{NaBH}_{4}, \mathrm{BF}_{3} \cdot \mathrm{Et}_{2} \mathrm{O}, \mathrm{THF}$, (iii) Jones reagent. (iv) Methanesulphonic acid, $\mathrm{P}_{2} \mathrm{O}_{5}$. 


\section{Conclusions}

The cyclopentane annulation strategy developed in our laboratory was utilized to convert the tetracyclic compound 2 to the hitherto unknown curved hexaquinane dione 3 . In view of limitations associated with cyclopentane annulation strategies, the methodology demonstrated here may find useful application in the synthesis of various natural and non-natural products having the cyclopentane rings fused together.

\section{Experimental Section}

General. Melting points were recorded on Labhosp or Veego melting point apparatus. Infrared (IR) spectra were recorded on Nicolet Impact-400 FT IR spectrometer. Solid samples were recorded as $\mathrm{KBr}$ wafers and liquid samples as film between $\mathrm{NaCl}$ plates. Ultraviolet spectra were recorded on Shimadzu UV-260 instruments. NMR spectra were recorded with Varian VXR 300 spectrometers $\left({ }^{1} \mathrm{H}\right.$ at $300 \mathrm{MHz},{ }^{13} \mathrm{C}$ NMR at $75.4 \mathrm{MHz}$ ) using $\mathrm{CDCl}_{3}$ or $\mathrm{CDCl}_{3} / \mathrm{CCl}_{4}$ solutions, and chemical shifts were referenced to TMS. Mass spectral measurements were carried out on GCD 1800 Hewlett-Packard GS-MS spectrometer. The high-resolution mass measurements were carried out using JEOL JMS-DX 303 GC-MS instrument. Accurate mass measurement by CI ionization method was carried out using Autospec X instrument. Elemental analysis was performed on Carlo-Erba MOD $1106 \mathrm{CHN}$ analyzer. Analytical thin-layer chromatography (TLC) was performed on glass plates $(10 \times 5 \mathrm{~cm})$ coated with Acme's silica gel G or GF 254 (containing 13\% calcium sulfate as a binder). Silica gel was coated on glass plate using the 'Sandwich Technique'. All reactions were monitored employing TLC technique using appropriate solvent system for development. Transfer of moisture sensitive materials were carried out in a glove box, using standard syringe-septum techniques, and the reactions were carried out under nitrogen or argon atmosphere until work up.

Compounds (4) and (5). The tetracyclic enone $2^{1}(525 \mathrm{mg}, 2.45 \mathrm{mmol})$ in ethyl acetate $(40 \mathrm{~mL})$ was treated with hydrogen in the presence of $10 \% \mathrm{Pd} / \mathrm{C}(24 \mathrm{mg})$ at $3 \mathrm{~atm}$ pressure for $4 \mathrm{~h}$. The reaction mixture was filtered and the solvent was removed under reduced pressure on a rotary evaporator. The mixture of products obtained was purified by chromatography (silica gel; petroleum ether/ethyl acetate 4:1) furnishing the minor saturated tetracyclic diketone 4 (85 $\mathrm{mg}$, $16 \%$ ) as off-white solid; $\mathrm{mp} 88-89{ }^{\circ} \mathrm{C} . R_{f} 0.62$ (ethyl acetate/petroleum ether 1:4). IR ( $\mathrm{KBr}$ ): $v_{\max } 2945,2917,2878,1726,1457,1175,1017 \mathrm{~cm}^{-1} .{ }^{1} \mathrm{H}$ NMR $\left(300 \mathrm{MHz}, \mathrm{CDCl}_{3}\right): \delta 3.26(1 \mathrm{H}, \mathrm{d}$, $J=9 \mathrm{~Hz}), 3.03(1 \mathrm{H}, \mathrm{t}, J=9 \mathrm{~Hz}), 1.43-2.82(14 \mathrm{H}, \mathrm{m}), 0.94-1.24(2 \mathrm{H}, \mathrm{m}) .{ }^{13} \mathrm{C}$ NMR $(75 \mathrm{MHz}$, $\left.\mathrm{CDCl}_{3} / \mathrm{CCl}_{4}\right): \delta 220.8,220.6,54.4,53.6,49.0,46.7,44.0,39.9,37.5,36.0,35.9,34.8,23.2,23.1$. Anal. calcd. for $\mathrm{C}_{14} \mathrm{H}_{18} \mathrm{O}_{2}$ : C, 77.03; H, 8.31. Found: C, 77.04; H, 8.60.

Continued elution of the column with petroleum ether/ethyl acetate (7:3) gave isomer $5(322 \mathrm{mg}$, $60 \%$ ) as off-white solid; $\mathrm{mp} 156-158{ }^{\circ} \mathrm{C} . R_{f} 0.32$ (ethyl acetate/petroleum ether 1:4). IR (KBr): 
$v_{\max } 2942,2874,1735,1458,1150,1091,947 \mathrm{~cm}^{-1} .{ }^{1} \mathrm{H}$ NMR $\left(300 \mathrm{MHz}, \mathrm{CDCl}_{3}\right): \delta 2.00-2.88$ $(14 \mathrm{H}, \mathrm{m}), 1.49-1.74(2 \mathrm{H}, \mathrm{m}), 0.87-0.98(2 \mathrm{H}, \mathrm{m}) .{ }^{13} \mathrm{C} \mathrm{NMR}\left(75 \mathrm{MHz}, \mathrm{CDCl}_{3} / \mathrm{CCl}_{4}\right): \delta 219.9$, 52.7, 50.3, 49.6, 44.2, 39.3, 39.1, 25.9. Anal. calcd. for $\mathrm{C}_{14} \mathrm{H}_{18} \mathrm{O}_{2}$ : C, 77.03; $\mathrm{H}, 8.31$. Found: C, 77.52; H, 8.19.

Compounds (6) and (7). To activated magnesium turnings (400 mg, $16.45 \mathrm{mmol}$ ), allyl bromide $(0.8 \mathrm{~mL}, 9.2 \mathrm{mmol})$ in dry ether $(5 \mathrm{~mL})$ was added at $0{ }^{\circ} \mathrm{C}$; stirring was continued at room temperature for $2 \mathrm{~h}$. To the Grignard reagent at $0{ }^{\circ} \mathrm{C}$ was then added a solution of dione $5(0.108$ $\mathrm{g}, 0.50 \mathrm{mmol})$ in dry THF $(25 \mathrm{~mL})$ and the mixture was stirred at room temperature for $2 \mathrm{~h}$. The reaction mixture was poured onto saturated ammonium chloride solution $(100 \mathrm{~mL})$, and the solvent was removed under reduced pressure on a rotary evaporator. The reaction mixture was extracted with ethyl acetate $(200 \mathrm{~mL})$ and the organic layer was washed with water $(100 \mathrm{~mL})$, brine $(100 \mathrm{~mL})$ and dried. Removal of the solvent on a rotary evaporator under reduced pressure gave a crdue product, which was separated and purified by column chromatography (silica gel, petroleum ether/ethyl acetate 9:1) furnishing the symmetric diol 6 (63 mg, 45\%) as a colorless liquid. $R_{f} 0.63$ (petroleum ether/ethyl acetate 1:2.3). IR (neat): $v_{\max } 3424,3075,2935,2864$, 1639, 1452, 1051, $911 \mathrm{~cm}^{-1}$. ${ }^{1} \mathrm{H}$ NMR $\left(300 \mathrm{MHz}, \mathrm{CDCl}_{3}\right): \delta 5.85-5.99(2 \mathrm{H}, \mathrm{m}), 5.08-5.19(4 \mathrm{H}$, $\mathrm{m}), 2.53-2.70(2 \mathrm{H}, \mathrm{m}), 2.25-2.38(4 \mathrm{H}, \mathrm{m}), 2.02-2.06(2 \mathrm{H}, \mathrm{m}), 1.56-1.78(10 \mathrm{H}, \mathrm{m}), 1.25-1.44$ $(4 \mathrm{H}, \mathrm{m}), 0.82-0.96(2 \mathrm{H}, \mathrm{m}) .{ }^{13} \mathrm{C} \mathrm{NMR}\left(75 \mathrm{MHz}, \mathrm{CDCl}_{3}\right): \delta 134.6,118.4,82.0,60.3,46.9,46.2$, 45.6, 43.9, 39.8, 38.2, 29.9. HRMS: calcd. for $\mathrm{C}_{20} \mathrm{H}_{30} \mathrm{O}_{2}-\mathrm{H}_{2} \mathrm{O}$ : 284.2140; found: 284.2137.

Continued elution of the column with the same solvent furnished the trans-annular diol 7 (43 mg, $30 \%$ ) as a white solid; $\mathrm{mp} 105-107^{\circ} \mathrm{C} . R_{f} 0.55$ (petroleum ether/ethyl acetate 1:2.3). IR (KBr): $v_{\max } 3373,3075,2930,2861,1643,1456,1153,1090,912 \mathrm{~cm}^{-1} .{ }^{1} \mathrm{H}$ NMR $\left(300 \mathrm{MHz}, \mathrm{CDCl}_{3}\right)$ : 5.90-6.04 (1H, m), 5.08-5.23 (2H, m), $2.97(1 \mathrm{H}, \mathrm{s}), 2.88(1 \mathrm{H}, \mathrm{s}), 2.21-2.85(7 \mathrm{H}, \mathrm{m}), 1.86-2.13$ $(4 \mathrm{H}, \mathrm{m}), 1.32-1.65(7 \mathrm{H}, \mathrm{m}), 1.05-1.28(1 \mathrm{H}, \mathrm{m}) .{ }^{13} \mathrm{C} \mathrm{NMR}\left(75 \mathrm{MHz}, \mathrm{CDCl}_{3}\right): 133.8,119.1,87.7$, 82.3, 55.7, 53.0, 50.3, 48.7, 47.2, 46.4, 44.3, 40.9, 39.6, 39.5, 37.4, 31.0, 29.4. HRMS: calcd. for $\mathrm{C}_{17} \mathrm{H}_{24} \mathrm{O}_{2}-\mathrm{H}_{2} \mathrm{O}: 242.1670$; found: 242.1669 .

Compound (8). To a stirred solution of diol $6(55 \mathrm{mg}, 0.18 \mathrm{mmol})$ and $\mathrm{NaBH}_{4}(120 \mathrm{mg}, 3.17$ $\mathrm{mmol})$ in dry THF $(30 \mathrm{~mL})$, freshly distilled $\mathrm{BF}_{3} . \mathrm{OEt}_{2}(0.7 \mathrm{~mL}, 5.69 \mathrm{mmol})$ in dry THF $(5 \mathrm{~mL})$ was added at $0{ }^{\circ} \mathrm{C}$ over a period of $30 \mathrm{~min}$. The reaction was stirred at room temperature for $1 \mathrm{~h}$ and then cooled to $0{ }^{\circ} \mathrm{C}$. Freshly prepared Jones reagent $(4 \mathrm{~mL})$ was added dropwise. The solution was stirred at room temperature for $4 \mathrm{~h}$, and THF was removed on a rotary evaporator under reduced pressure. The reaction mixture was extracted with ethyl acetate $(150 \mathrm{~mL})$, the organic layer was washed with water $(25 \mathrm{~mL})$, brine $(25 \mathrm{~mL})$, and dried. Removal of the solvent gave the crude lactone $\mathbf{8}$, which was purified by column chromatography (silica gel, hexane/ethyl acetate $1: 1)$ to furnish pure lactone $8(40 \mathrm{mg}, 66 \%)$ as a white solid; mp $123-124{ }^{\circ} \mathrm{C} . R_{f} 0.53$ (petroleum ether/ethyl acetate 1:4). IR (KBr): $v_{\max } 2925,2855,1755,1459,1421,1167,921 \mathrm{~cm}^{-}$ ${ }^{1} .{ }^{1} \mathrm{H}$ NMR $\left(300 \mathrm{MHz}, \mathrm{CDCl}_{3}\right): \delta 2.53-2.72(4 \mathrm{H}, \mathrm{m}), 2.12-2.22(2 \mathrm{H}, \mathrm{m}), 2.04-2.08(4 \mathrm{H}, \mathrm{m})$, $1.60-1.81(2 \mathrm{H}, \mathrm{m}), 1.25-1.58(14 \mathrm{H}, \mathrm{m}) .{ }^{13} \mathrm{C} \mathrm{NMR}\left(75 \mathrm{MHz}, \mathrm{CDCl}_{3} / \mathrm{CCl}_{4}\right): \delta 176.6,94.4,59.7$, $48.8,46.5,42.5,38.4,37.8,33.1,30.1,29.0$. MS [GC-MS]: $m / z 294\left(\left[\mathrm{M}-2 \mathrm{H}_{2} \mathrm{O}\right]^{+}\right)$. 
Compound (9). To a stirred solution of diol 7 (35 mg, $0.1346 \mathrm{mmol})$ and $\mathrm{NaBH}_{4}(72 \mathrm{mg}, 1.9$ $\mathrm{mmol})$ in dry THF $(25 \mathrm{~mL})$, freshly distilled $\mathrm{BF}_{3} \cdot \mathrm{OEt}_{2}(0.4 \mathrm{~mL}, 3.25 \mathrm{mmol})$ in dry THF $(5 \mathrm{~mL})$ was added at $0{ }^{\circ} \mathrm{C}$ over a period of $45 \mathrm{~min}$. The reaction was stirred at room temperature for $2 \mathrm{~h}$ and then cooled to $0{ }^{\circ} \mathrm{C}$. Freshly prepared Jones reagent $(5 \mathrm{~mL})$ was added dropwise. The solution was stirred at room temperature for $3 \mathrm{~h}$, and then THF was removed on a rotary evaporator under reduced pressure. The reaction mixture was extracted with ethyl acetate (150 $\mathrm{mL})$ and the organic extract was washed with water $(50 \mathrm{~mL})$, brine $(50 \mathrm{~mL})$, and dried. Removal of the solvent gave the crude lactone $\mathbf{9}$, which was purified by column chromatography (silica gel, petroleum ether/ethyl acetate 2:3) to furnish pure lactone 9 (20 mg, 54\%) as a white solid; mp 79-80 ${ }^{\circ} \mathrm{C} . R_{f} 0.37$ (ethyl acetate). IR (KBr): $v_{\max } 2928,2859,1759,1456,1108,1047,912$ $\mathrm{cm}^{-1} .{ }^{1} \mathrm{H}$ NMR $\left(300 \mathrm{MHz}, \mathrm{CDCl}_{3}\right): \delta 3.26(1 \mathrm{H}, \mathrm{bs}), 2.80-2.86(1 \mathrm{H}, \mathrm{m}), 2.01-2.63(15 \mathrm{H}, \mathrm{m})$, 1.13-1.25 (5H, m). ${ }^{13} \mathrm{C}$ NMR $\left(75 \mathrm{MHz}, \mathrm{CDCl}_{3} / \mathrm{CCl}_{4}\right): \delta 176.0,150.7,122.5,94.0,52.0,51.6$, $50.4,46.5,45.1,45.0,44.0,42.8,41.7,40.0,39.9,32.4,28.3$. MS [GC-MS]: $m / z 256$ ([M$\left.\mathrm{H}_{2} \mathrm{O}\right]^{+}$).

Compound (3). Lactone 8 (25 mg, $0.07 \mathrm{mmol})$ was dissolved in methanesulphonic acid (5 mL) and $\mathrm{P}_{2} \mathrm{O}_{5}$ (approximately $10 \mathrm{mg}$ ) was added. The reaction mixture was heated at $50-55{ }^{\circ} \mathrm{C}$ for 4 $\mathrm{h}$, cooled to $0{ }^{\circ} \mathrm{C}$, and diluted with water $(50 \mathrm{~mL})$. The reaction mixture was extracted with chloroform $(100 \mathrm{~mL})$, and the organic layer was washed with saturated sodium bicarbonate solution $(75 \mathrm{~mL})$, brine $(75 \mathrm{~mL})$, and dried. Evaporation of the solvent at reduced pressure gave the crude hexacyclic enone 3 , which was purified by column chromatography (silica gel, petroleum ether/ethyl acetate $7: 3)$ to furnish dione $3(12 \mathrm{mg}, 54 \%)$ as a white solid; $\mathrm{mp} 134{ }^{\circ} \mathrm{C}$. $R_{f} 0.43$ (ethyl acetate). IR (KBr): $v_{\max } 2926,2857,1700,1630,1458,1351,1173,1026 \mathrm{~cm}^{-1}$. UV $\left(\mathrm{CH}_{3} \mathrm{OH}\right): \lambda_{\max }(\varepsilon) 246.4 \mathrm{~nm}(3,107) .{ }^{1} \mathrm{H} \mathrm{NMR}\left(400 \mathrm{MHz}, \mathrm{CDCl}_{3}\right): \delta 3.34-3.50(2 \mathrm{H}, \mathrm{m}), 3.08-$ $3.16(2 \mathrm{H}, \mathrm{m}), 2.72-2.82(4 \mathrm{H}, \mathrm{m}), 2.46-2.66(8 \mathrm{H}, \mathrm{m}), 2.14-2.24(1 \mathrm{H}, \mathrm{m}), 2.02-2.12(2 \mathrm{H}, \mathrm{m})$, 1.80-1.94 (2H, m), 1.48-1.60 (1H, m). ${ }^{13} \mathrm{C}$ NMR (75 MHz, $\left.\mathrm{CDCl}_{3}\right): \delta 204.4,187.1,147.1,57.3$, 52.4, 47.9, 46.4, 41.0, 38.6, 31.9, 24.3. HRMS: calcd. for $\mathrm{C}_{20} \mathrm{H}_{22} \mathrm{O}_{2}$ : 294.1619; Found: 294.1616.

\section{Acknowledgements}

The authors thank CSIR for financial support and SAIF-Mumbai for providing the spectral data. RS thanks CSIR for the fellowship. We thank Prof. A. Srikrishna, IISc, Bangalore for providing NMR data for some of the intermediates. SK thanks DST for the award of J. C. Bose fellowship.

\section{References}

1. (a) Kotha, S.; Brahmachary, E.; Sivakumar, R.; Joseph, A.; Sreenivasachary, N. Tetrahedron Lett. 1997, 38, 4497. (b) Kotha, S.; Sivakumar, R. Arkivoc 2005, (xi), 78. 
2. (a) Kotha, S.; Sivakumar, R.; Damodharan, L.; Pattabhi, V. Tetrahedron Lett. 2002, 43, 4523.

3. (a) Bertz, S. H.; Cook, J. M.; Gawish, A.; Weiss, U. Org. Syn. Coll. 1990, (vii), 50. (b) Gupta, A. K.; Fu, X.; Synder, J. P.; Cook, J. M. Tetrahedron 1991, 47, 3665. (c) Fu, X.; Cook, J. M. Aldrichim Acta 1992, 25, 43.

4. Sivakumar, R. Ph.D. Thesis, IIT-Bombay, 2001. 